\title{
Migraciones subsharianas a Argentina: interacción, integración o exclusión
}

\author{
Sub-saharan Migrations to Argentina: Interaction, \\ Integration or Exclusion
}

\section{Migrações subsaarianas a Argentina: interação, integração ou exclusão}

\section{Gisselle Kleidermaster* • Argentina}

Recibido el 19 de abril de 2011, aceptado el 6 de diciembre de 2011

\section{Resumen}

Objetivo: indagar por las representaciones que la población nativa de tres barrios de Buenos Aires (Flores, Once y Constitución) tiene acerca de los nuevos inmigrantes del África Subshariana, así como una posible correspondencia entre clase social y racismo, vinculando este hecho a la historia de la conformación del Estado-nación Argentino. Metodología: investigación cualitativa, basada en observación participante y entrevistas semi-estructuradas realizadas a población inmigrante de países subsaharianos, así como también, a población "nativa" (argentinos, principalmente empleados de comercio y vendedores ambulantes) con los cuales mantienen interacciones cotidianas en el barrio de Flores, polo comercial de la Capital Federal, Provincia de Buenos Aires. Resultados: se observa la existencia de un discurso "políticamente correcto" pero que presenta elementos fuertemente discriminatorios al indagar más en profundidad sobre ciertos aspectos que refieren al "control" de la población migrante. Conclusiones: el estudio demuestra que efectivamente hay espacios de conflicto donde el migrante "molesta", resulta "extraño", no sólo por su condición nacional, sino también por su condición racial.

Palabras claves: políticaMigración - África Subsahariana - Argentina-Discriminación- Racismo.

* Por migraciones subsaharianas se entiende la llegada de población a la Argentina durante la última década del siglo XX y primera del siglo XXI, provenientes de países del continente africano situados al sur del Sahara, principalmente Senegal, Gambia, Ghana, Malí, Sierra Leona y Nigeria. Este documento se basa en un trabajo de investigación que se realiza como parte de tesis doctoral, en la Universidad de Buenos Aires, bajo el título "La discriminación y el racismo en Argentina: El caso de los inmigrantes africanos y los afrodescendientes". La misma cuenta con financiamiento de Conicet (Consejo Nacional de Ciencia y Tecnología).

** Argentina. Socióloga. Doctora en Ciencias Sociales. IIGG-UBA-CONICET Argentina. kleidermacher@gmail.com 


\begin{abstract}
Objective: this paper aims at investigating the representations of the population of three neighborhoods of Buenos Aires (Flores, Once and Constitución) about the new immigrants from Sub-Saharan Africa and a possible correlation between social class and racism, relating this fact to the history of the making of the Argentine nation-state. Methodology: it is a qualitative research based on participant observation and semistructured interviews to sub-Saharan immigrant population, as well as to Argentinian people (Argentinians, mainly sellers and informal vendors), with whom they have daily interactions in the neighborhood of Flores, commercial pole of the Federal Capital, Buenos Aires. Results: it is observed the existence of a "politically correct" discourse, but with strong discriminatory elements when inquiring more deeply about certain issues related to the "control" of the migrant population. Conclusions: the study shows that there are indeed areas of conflict where migrants "disturb", become "strange", not only for their national origin, but also for their racial status.
\end{abstract}

Keywords: migration policy, Sub-saharan Africa, Argentina, discrimination, racism.

\title{
Resumo
}

Objetivo: indagar pelas representações que a povoação nativa de três bairros de Buenos Aires (Flores, Onze e Constituição) tem cerca dos novos imigrantes de África Subsaariana, assim como uma possível correspondência entre classe social e racismo, vinculado este fato à historia da conformação do Estado -nação Argentino. Metodologia: pesquisa qualitativa, baseada em observação participante e entrevistas semi-estruturadas realizadas a povoação imigrante de países subsaarianos, assim como também, a povoação "nativa" ( argentinos, principalmente empregados) de comercio e vendedores ambulantes) com os quais mentem interações cotidianas no bairro de Flores, pólo comercial da Capital Federal, Província de Buenos Aires. Resultados: observa se a existência de um discurso "politicamente correto", mas que apresenta elementos fortemente discriminatórios ao indagar mais em profundidade sobre certos aspectos que referem ao "controle" da povoação migrante. Conclusões: a pesquisa demonstra que efetivamente há espaços de conflito onde o migrante "molesta", resulta "estranho", não só por sua condição nacional, também por sua condição racial.

Palavras-Chaves: política de migração, África subsaariana, Argentina, discriminação, racismo. 


\section{Introducción}

El presente trabajo se estructura en dos secciones: la primera de índole teórica, en la que se realiza una revisión de diversos trabajos fundantes de los estudios sobre la población afro en Argentina. A través de los mismos, se pueden analizar las diversas políticas que formaron parte del diseño de Argentina independentista, basadas en las teorías existentes en Europa que fueron retomadas en las Américas, en un primer momento como modo de legitimar la colonización, luego la esclavitud, y en la etapa de construcción del Estado-nación, como un modo de homogeneizar la población tras la ilusión de una nación homogénea, blanca, europea y católica. Esta revisión permitirá obtener herramientas teóricas para analizar los fundamentos sobre los que se construyó el Estado-Nación argentino, y sus ideales de nación blanca, homogénea y católica.

En la segunda parte, con base en las formulaciones teórico-empíricas delineadas en la primera, se analiza el corpus empírico recogido en el trabajo de campo sustentado en las entrevistas semi estructuradas y la observación participante en el barrio de Flores ${ }^{1}$. La elección de dicho barrio se fundamenta en la accesibilidad, la presencia de vendedores ambulantes procedentes de África Subsahariana producto de la inmigración reciente, así como la gran cantidad de interacciones que con ellos se generan por ser este barrio un importante polo comercial de la Capital Federal.

- ¿A qué se refiere la alusión al Racismo en este estudio?

La concepción tradicional de racismo se ha referido históricamente a un conjunto de creencias y actitudes relativamente coherentes y discriminatorias con respecto a un grupo humano identificado por una serie de atributos físicos heredados biológicamente. Se trata de cogniciones, acciones y procedimientos que contribuyen al desarrollo y la perpetuación de un sistema en el cual los blancos dominan a los negros (Essed, 1991, p. 132).

Sin embargo, una de las características a las que refiere la moderna conceptualización del racismo es precisamente que para que una ideología racista prospere, no es necesario que exista una raza. De hecho, la biología humana asegura que las razas no existen. En efecto, la ideología racista crea la raza al identificar como tal al grupo o grupos que se convierten en objeto de su atención (Stavenhagen, 2000).

Es por eso que hoy el concepto Raza está dejando paso al de Etnia porque permite disuadir de las pesadas implicaciones que aquel concepto ha tenido; es decir, a las implicaciones ligadas a las doctrinas racistas y a los regímenes políticos que implantaron una política racial. Como indica Colette (1992), ya sea que se utilice la expresión etnia o raza, se trata de nombrar a grupos que presentan rasgos específicos diferenciados:

Se trata de un polo comercial de la Capital Federal (Buenos Aires, Argentina) 
una lengua común, una historia compartida, (o el mito de tal historia), una religión, una nación de origen (o un mito nacional), etc.

No obstante, cualquiera que sea el término usado para designar los grupos, la aprehensión ideológica sigue estableciendo grupos provistos de una esencia propia, productora de conductas y de cualidades específicas inscritas en la carne, y la sangre. Hay varias definiciones de racismo que toman varios de estos conceptos (ideología, prácticas, conjunto de pre-conceptos, que identifican a una población "diferente" por distintos rasgos que los marcan como "otros”), pero lo que tienen en común es el pensarlo como una justificación de los sistemas de dominación impuestos por la Europa Colonial, y luego, Imperial, al resto del mundo.

En este sentido, este estudio se basa en el modelo propuesto por Guillaumin (1992) en el que identifica tres fases que puede tomar el racismo en los Estados Modernos, en las que, a la manera de etapas, una incluye a la antecesora, lo cual brinda un marco para comprender la manera en que se construyó la idea del "otro" en Argentina, y, por lo tanto, ofrece más herramientas para analizar el caso de la población afro en este país.

La primera etapa se enmarca en la ideología cotidiana, en la presunción de homogeneidad del grupo comunitario o nacional. Tal como fue planteado en el apartado anterior, la idea de que un mismo cuerpo formaría al "pueblo", es decir, de la misma naturaleza que su cultura, su historia, su mentalidad, sus gustos. Se trata del mito nacional que inscribe la idea de que todos los argentinos, sea cual fuere su color, son y siempre son "blancos" y por eso todos son "buenos" y "apreciables"1.

La segunda, se enmarca en la práctica militante y política: la explicación de la idea de la diferencia, la "demostración científica" que intenta establecer una cadena causal en esos mismos fenómenos y procede por lo tanto a una separación e identificación de los elementos cuestionados, sería el nivel de "porque".

De este hecho se pueden desprender varios elementos de análisis: en primer lugar, como ya se dijo, la justificación de las desigualdades, es decir, la población afroargentina, como producto de la esclavitud y todas las argumentaciones que se brindaron para justificarla, quedó estigmatizada como un elemento inferior y menospreciado. Para sus descendientes, la esfera que les queda reservada como espacio de dominación no pertenece ni al poder, ni a la ciencia, ni a la literatura.

En este sentido, María Elena Vela (2001) observa que en la actualidad, habría un imaginario social contemporáneo que recupera una parte importante de las visiones

1 Como analiza Frigerio (2006) la categorización de una persona como "no negra" se produce a través de un trabajo de construcción social de la realidad de constante invisibilización de los rasgos fenotípicos negros a nivel micro. Esta invisibilización a nivel de las interacciones micro sociales, se corresponde a nivel macro con la invisibilización de la presencia del negro en la historia argentina y de sus influencias a la cultura. 
forjadas en siglos anteriores sobre los africanos, donde eran visualizados principalmente como sirvientes de los blancos y presentados como brutales, poco confiables, taimados y con propensión a engañar a sus empleadores, además de ser sucios, podían oler mal y se consideraban poco trabajadores y propensos a la diversión. De aquel pasado proviene aquel uso de "negro" como sinónimo de "malo" y "despreciable".

Por último, la tercera etapa corresponde a las instituciones del Estado; esta es la expresión más formalizada de la ideología racista, el sistema jurídico, la doctrina política, cuyo objetivo es disponer del Estado. Esta prescribe la conducta que se supone que deriva del conocimiento de los hechos, sería el nivel del "por lo tanto". El orden debe reinar conforme a divisiones que serían naturales.

Sin embargo, en Argentina, la legislación no impone formas evidentes de discriminación; técnicamente todos los ciudadanos son iguales ante la ley, no hay normas formalizadas que impongan la distribución social o espacial o indiquen que algunas clases de habitantes tienen menores derechos. Esto no quiere decir que no exista racismo, sino que se manifiesta de otras maneras no tan visibles.

Hoy el racismo concebido por la ideología nacional centra su atención en las diferencias culturales, alegando la incapacidad de las comunidades inmigrantes de formar parte de la nación (Stavenhagen, 2000). Pero también hay que tener en cuenta que en la Argentina, al contrario que en Norteamérica, el principal discurso para explicar las desigualdades sociales es la clase, así la raza ha permanecido poco enunciable, los modelos explicativos son mucho más implícitos que explícitos.

La clasificación y la identificación racial de la mayor parte de los individuos varían de acuerdo con su situación socio-económica, el contexto en que se hallan y la propia construcción identitaria. Dado que una gota de sangre blanca permite clasificar a un individuo como tal, es probable que en su mayoría fueran considerados "no negros".

Como menciona Margulis (1999), a través de la racialización de las relaciones de clase, estos grupos se desarrollan socialmente dentro de un sistema dado de relaciones económicas y políticas donde, a partir de sus rasgos corporales (color de la piel, mestizaje), están ubicados en condiciones desfavorables en las relaciones económicas (trabajos penosos, poco estables, mal remunerados) y la combinación histórica de estos rasgos, sumado a su ubicación espacial (periférica, barrios marginados), y social (pobreza, desempleo) producto de las mismas condiciones económicas y sociales, conlleva desventajas en cuanto a sus posibilidades de logros en el plano de la educación y de otros ámbitos/espacios sociales apreciados.

Por lo tanto, la pobreza está asociada en el imaginario porteño con la negritud. Lo que demuestra la efectiva vigencia de las categorías raciales como reproductoras de las

1 En la actualidad, el término Negro suele ser antecedido o precedido por otros términos para denotar un insulto. 
diferencias sociales que nos aquejan, a través de la insistencia en que la categoría "negro" o "cabecita negra" asignada a buena parte de la población de escasos recursos no involucra una dimensión racial sino meramente socio-económica

Se puede entonces argumentar, desde un punto de vista macro, el racismo es a la vez un sistema de desigualdades estructurales y un proceso histórico que se crean y se recrean a través de prácticas rutinarias. Como sintetiza Essed (1991) se trata de un conjunto de relaciones sociales que se reproducen entre los individuos y los grupos y se encuentran organizadas como prácticas sociales. Por ello, la imputación de responsabilidad, no sólo es en el caso de acción, sino también de inacción. Un grave problema en la actualidad es la inacción que impera en el grupo dominante y en particular la tolerancia pasiva del racismo. Por eso, si bien no hay leyes que impongan diferencias de derechos y obligaciones a la población del país según sus rasgos físicos, el hecho de no impedir esta jerarquización social que está implícita en la sociedad, puede ser considerada también una forma de racismo.

\section{Breves observaciones sobre las ideologías racistas en la Argentina colonial}

Para analizar los procesos discriminatorios actuales, hay que remontarse a la historia y descubrir algunos antecedentes lejanos que son comunes a diversos países de América Latina, en especial a Argentina, y que tienen que ver con la historia de colonización.

Como analiza Morner (1970), la sociedad de castas de Hispanoamérica fue creada transfiriendo al Nuevo Mundo la forma corporativa y jerárquica, basada en el Patrimonio de Castilla de la Edad Media tardía, e imponiéndola a una situación colonial multirracial. Esta realidad transatlántica se caracterizaba por la dicotomía de conquistadores y conquistados, amos y siervos o esclavos, y, en segundo término, por el cruzamiento entre estos grupos opuestos. Por lo tanto, resultaba inevitable que la estratificación social y el estatus social se relacionaran estrechamente con la división en grupos étnicos y la ubicación de los mismos dentro de la estructura social jerárquica, lo que dio lugar al surgimiento de lo que el estudioso chileno Lipschutz (1944) llamó ingeniosamente "pigmentocracia" (Citado en Morner, 1970, p. 61).

Este proceso de jerarquización de la población puede ser entendido, si se sigue la línea de pensamiento de Aníbal Quijano (2000), como una forma de otorgar legitimidad a las relaciones de dominación impuestas por la conquista. Los pueblos conquistados y dominados fueron situados en una posición natural de inferioridad y, en consecuencia, también sus rasgos fenotípicos, así como sus descubrimientos científicos y culturales. De ese modo, la raza se convirtió en el primer criterio fundamental para la distribución de la población mundial en los rangos, lugares y roles en la estructura de poder de la nueva sociedad (Quijano, 2000).

Para citar este artículo:
Kleidermaster, G. (junio, 2012). Migraciones subsharianas a Argentina: interacción, integración o exclusión. Ánfora, 19 (32), 91-116. Universidad Autónoma de Manizales. ISSN 0121-6538. 
El poder colonial hizo que todos los pueblos fueran despojados de sus propias y singulares identidades históricas para tener una nueva identidad racial, colonial y negativa, lo que implicaba el despojo de su lugar en la historia de la producción cultural de la humanidad. Se trataba de razas inferiores, capaces sólo de producir culturas inferiores.

Esta situación ha sido caracterizada por Taguieff (2002) como una manera de justificar la opresión, es decir, cuando los hombres oprimen a sus semejantes, el opresor siempre encuentra, en el carácter del dominado, la justificación de su opresión. Las alegaciones más comunes contra el oprimido son su ignorancia y su depravación, así como su impotencia para elevarse desde la decadencia hasta la civilización y respetabilidad.

Para el caso Argentino, Rodríguez (1961) observa que los españoles, a fin de justificar la esclavitud, ubicaban a los negros en las categorías de "traidores", "ociosos", "borrachos". Ese imaginario de la época colonial es una herencia que aún hoy perdura. Aunque se niegue la existencia de la distinción de razas, en la sociedad colonial, el negro era segregado y era considerado un mero objeto de trabajo, un ser inferior; esa actitud se proyectaba al mulato y al mestizo.

Esta atribución de determinadas cualidades, siempre negativas y peyorativas, siguiendo el análisis de Taguieff (2002), se debe a que, frente a las prácticas de explotación, como las ocurridas fundamentalmente durante el período que analizamos, los explotadores deben justificar y justificarse argumentativamente la situación de dominación, y el argumento más utilizado se fundamentó en la teoría de la evolución o la deshumanización; es decir, en racionalizar los prejuicios y estereotipos de los "otros" naturalizando ciertos atributos negativos de la "raza" deshumanizada, mediante una proyección de las consecuencias de la explotación racializante sobre la naturaleza específica de dicha "raza".

\section{Algunas consideraciones sobre la migración africana hacia Argentina}

Dado que el trabajo se concentra en la población africana, es necesario realizar un breve repaso sobre su presencia en el territorio Argentina. Desde 1510 la corte de España vio que, para compensar la falta de población de las provincias conquistadas entonces en América, no había otro medio sino trasladar hacia dicho continente nueva mano de obra proveniente de África, cuya trata regular comenzó en 1516.

El primer permiso real para importar esclavos en la región del Río de la Plata fue otorgado en 1534, dos años antes de la primera fundación de Buenos Aires; sin embargo, es en 1778 que el tráfico de esclavos entró en una fase de esplendor en Buenos

Para citar este artículo:
Kleidermaster, G. (junio, 2012). Migraciones subsharianas a Argentina: interacción, integración o exclusión. Ánfora, 19 (32), 91-116. Universidad Autónoma de Manizales. ISSN 0121-6538. 
Aires tras la liberalización de los puertos coloniales a los comerciantes extranjeros (Scheuss de Studer, 1958).

Es difícil determinar exactamente cuántos esclavos ingresaron a esta ciudad durante el período colonial, ya que las estadísticas sugieren que el volumen del comercio legal escasamente podía compararse con el ilegal ${ }^{1}$. Sin embargo, Goldberg (1976) afirma que era una población digna de ser considerada por su dimensión: en el padrón levantado en 1778 constituían casi el 30\% de la población, todos ellos traídos al puerto por los tratantes de esclavos desde África. Esta inmigración forzada de africanos se estima en 45.000 individuos que habrían cambiado el perfil de la ciudad entre 1740 y 1810.

Como afirma la citada historiadora, la población africana y afrodescendiente habría tenido en los censos y padrones de la ciudad y la campaña mucha mayor relevancia de la que usualmente se les ha concedido y estos datos deben haber sido aún menores que los reales, ya que la revelación de fuentes no censales hace pensar que deben haber estado sub-representados en dichos registros ${ }^{2}$.

Según los relatos de la época había una gran dependencia de Buenos Aires hacia sus trabajadores esclavos, en el sentido de que la fuerza laboral esclava formaba la base de la pirámide laboral de la ciudad. Pero esto revela el proceso discriminatorio de selección de empleos que tenía lugar en el Buenos Aires de 1780-1850. Los empleos menos deseables, más degradantes, más insanos y peor pagados estaban reservados para los afroargentinos. Como afirma Reid Andrews (1989) "era raro el hombre de color que podía esperar elevarse por encima del nivel de un artesano moderadamente exitoso, así como era rara la mujer de color que pudiera esperar más de la vida que una cómoda posición como confiable ama de llaves."

Como los negros estaban destinados a las ocupaciones más despreciables, la baja condición racial acordada a los afroargentinos se reforzaba mutuamente en un círculo que se tornaba imposible de quebrar. Una consecuencia de este proceso fue que la estructura ocupacional de los afroargentinos libres tendía a ser idéntica a la de los esclavos. Solo cambiaba la posición jurídica, pero no su condición económica ni su condición marginal en la sociedad.

Este proceso es lo que De Rudder y Poiret (2000) han llamado etnicización: se trata de la clasificación social y posicionamiento en una escala que ordena estatus sociales, económicos o políticos. En el transcurso de la "etnicización”, la atribución o la reivindicación de pertenecías étnicas se vuelve un referente determinante de la acción y la interacción y por lo tanto limita cualquier movilidad social.

1 Reid Andrews (1989) ejemplifica que de los 12.778 esclavos ingresados en Buenos Aires desde Brasil entre 1606 y 1625 solo 288 lo hicieron bajo permiso real.

2 Goldberg, Marta.(1976). La población negra y mulata de la ciudad de Buenos Aires, 1810-1840. Desarrollo Económico, 61(16):75-99.

Para
Kleidermaster, G. (junio, 2012). Migraciones subsharianas a Argentina: interacción, integración o exclusión. Ánfora, 19 (32), 91-116. Universidad Autónoma de Manizales. ISSN 0121-6538. 


\section{La construcción del Estado-nación "Blanco"}

Al finalizar el período colonial, los países sudamericanos se enfrentaban a la construcción del Estado (como aparato administrativo político) y de la Nación (como estructura ideológica-simbólica). Para ello se inició un proceso de homogeneización de los miembros de la sociedad mediante la eliminación y exclusión de una parte de la población que, siguiendo la perspectiva colonial, fueron los negros, indios y mestizos ${ }^{1}$ (Quijano, 2000, Geler, 2004).

Las categorías y clasificaciones, instaladas profundamente en la cultura, no solamente siguieron vigentes, sino que fueron reforzadas por nuevos aportes que acentuaron el racismo y la discriminación. Entre ellos están la Revolución Francesa y la expansión imperial que articuló y difundió un discurso racista que tendía a justificar la intromisión y el uso de la fuerza para invadir y dominar a muchos países en diversos continentes. El eje de ese discurso era la primacía del hombre blanco (Quijano, 2000). Para el caso de la Argentina, el ideal de una población blanca se manifestó en el prolongado esfuerzo por blanquear estadísticamente a nuestra población para disimular la incidencia de negros, indios y mulatos y negar a la población mestiza. Es decir, que se pasó de una sociedad altamente "racializada" donde los afroporteños ocupaban los peores empleos y eran muchas veces segregados de los lugares públicos, a una negación de dicha población. A continuación se realiza un breve repaso de dicho proceso en base a trabajos de diversos antropólogos e historiadores que han trabajado el tema (Rodríguez, 1961; Reid, 1989; Vela 1999; Frigerio, 2006).

Si bien los negros (esclavos, libertos y libres), los pardos y los morenos, habían constituido en el siglo XIX una parte significativa de la población de Buenos Aires, tanto por su número como por la variedad de actividades que desarrollaban, no "aparecieron" en la vida política y social del país sino en la época de Rosas² (Vela, 1999).

1 Como plantea Balibar (1988) El problema fundamental es producir el pueblo, mejor aún, que el pueblo se produzca a sí mismo en forma permanente de comunidad nacional. En sus propias palabras, "producir el efecto de unidad mediante el cual el pueblo aparecerá a los ojos de todos "como un pueblo", es decir, como la base y el origen del poder político" (1988:146)

2 Juan Manuel de Rosas fue Gobernador de Buenos Aires en los períodos 1829-1832 y 1835-1852. Entre 1820 y 1852, diversos grupos sociales con proyectos políticos diferentes se enfrentaron en los intentos por constituir un Estado en las Provincias Unidas del Río de la Plata. La diferencia entre los proyectos enfrentados tenía que ver con la forma de organización política que se quería dar, unos el centralismo y otros el federalismo. Los federales se oponían a un régimen de gobierno unitario en defensa de las autonomías provinciales. Pero en la provincia de Buenos Aires, la defensa de la autonomía provincial se transformó en una justificación para no ceder la ciudad y el puerto de Buenos Aires a un Estado central.

Desde 1828, el autonomismo de Buenos Aires se fue identificando cada vez más con Juan Manuel de Rosas - representante de los intereses de los hacendados y terratenientes de la provincia-.Los gobiernos federales de las provincias del Litoral y del interior siguieron reclamando al gobierno federal de Buenos Aires la libre navegación de los ríos y aranceles de aduana que protegieran sus industrias locales. 
Hasta entonces habían sido simplemente aptos para tareas serviles y secundarias en la vida civil y militar, y no considerados como sujetos históricos. Sólo se ocupaban de ellos las disposiciones que regulaban su vida, sus actividades y sus organizaciones, para que ninguna de ellas perturbara el orden y la tranquilidad de una comunidad creada por y para "la gente decente".

Los liberales argentinos que admiraban la formidable expansión económica, social, territorial y cultural lograda por los principales países europeos y los Estados Unidos, comenzaron a "enriquecerse" con las aportaciones de reconocidos pensadores suyos, que preconizaban el "racismo científico", como Gobineau y Chamberlain, quienes sostenían que los grandes éxitos de las principales naciones europeas se debían específicamente a la superioridad de la herencia genética de la población, que era predominantemente blanca.

En este sentido, Sarmiento (1995), considerado uno de los padres de la patria, expresaba:

Barbarie es lo heredado, las razas que se han mestizado han dado lugar a hombres aún más serviles. Civilización es lo que otros hombres están creando en el planeta. Aquello que debe ser apropiado por los hombres de la América de origen hispano. Cambiar la mente, cambiar la sangre, ha de ser la preocupación central de los nuevos emancipadores. Su civilización se considera a sí misma como civilizadora. Lucha entre la parte civilizada de las ciudades y la parte bárbara de las campañas. La lucha parecía política y era social ${ }^{1}$.

En Argentina, estas versiones se combinaron con los viejos resentimientos racistas de los unitarios exiliados por Rosas 2 , "y sus negros", para achacar a la mezcla de la escasa población blanca total con africanos e indios, la culpa del papel subordinado que desempeñaba la nación en el concierto mundial. Por tanto, la "generación del 80" se propuso poner en práctica un ingreso masivo de inmigrantes europeos ${ }^{3}$. Lo que deseaban bajo el lema de "gobernar es poblar" era modificar la estructura social modifican-

1 Domingo Faustino Sarmiento (1811-1888) publica en 1883 su obra "Conflictos y Armonías de las Razas en América".

2 Rosas reconoció la conveniencia de organizar las masas a su favor y dirigió especial atención a la población de color, "empleando una hábil combinación de propaganda, halagos y genuinas concesiones para granjearse el apoyo a su causa" Reid Andrews, 1989, p. 117). En otra cita el historiador observa "hasta el mismo fin, el apoyo de los afroargentinos a Rosas parece haber sido firme. Los negros de la ciudad exhibían de manera prominente las insignias federales rojas y lealmente se disponían a servir en los ejércitos de Rosas. Los candombes infaliblemente comenzaban con varios versos en honor del gobernador escritos por los negros mismos o por propagandistas de Rosas" (1989, p. 119).

3 Bajo la denominación de la Generación del 80 se conoce a una élite gobernante de la República Argentina durante el crucial período de 1880-1916. Procedentes de familias ricas de las provincias y de la capital, se nuclearon en el Partido Autonomista Nacional, fusión de las fuerzas dominantes en el período precedente, el Partido Autonomista y el Partido Nacional, ambos sucesores de la disgregación del antiguo Partido Unitario. En 1880, lanzaron la candidatura a la presidencia de 
do su composición étnica. Esto implicó que la población negra, no sólo fue fagocitada por la gran oleada de inmigrantes europeos, sino que igual proceso sufrió en la misma historia fundacional contribuyendo de esta manera a crear el mito de que en Argentina nunca hubo negros ${ }^{1}$ (Vela 1999, 2001; Geler 2007, 2010).

Como plantea Balibar (1988), el mito de los orígenes y la continuidad nacionales es una forma ideológica efectiva en la que se construye cotidianamente la singularidad imaginaria de las formaciones nacionales, remontándose desde el presente hacia el pasado. Lo característico de los Estados, sean cuales fueren, es representar el orden que instituyen como eterno, pero la práctica demuestra que lo que ocurre es más o menos lo contrario. Ninguna nación moderna posee una base "étnica" dada, aunque proceda de una lucha de independencia nacional.

Sin embargo, y hasta fechas recientes, los argentinos se han visto a sí mismos como un pueblo homogéneamente blanco, construido a partir del aporte de una variedad de raíces europeas armónicamente integradas en una unidad nacional. La creación de este "mito" puede datarse a partir del Censo Nacional de 1895 que estableció que más del $80 \%$ de la población del país era de "raza" blanca y origen europeo.

En cuanto al debilitado resto integrado por unos pocos indígenas y descendientes de africanos, dada la acelerada tasa de blanqueamiento no se dudaba de que estaban abocados a la pronta desaparición total, por lo que el censo afirmaba que la cuestión de las razas tan importante en los Estados Unidos, ya no existía en la Argentina" (Quijada, 2004, p. 206).

Por más de un siglo se extiende la historia de la negación de la presencia de los africanos en el territorio argentino. Para fundamentar el mito de su desaparición, se construyeron diversos ejes argumentales, que si bien tienen correlato con la realidad,

Julio Argentino Roca, general que había dirigido la Conquista del Desierto un año antes, y que fue el artífice de la generación y del modelo de país que ésta representó. En lo social, abogaron por los cánones positivistas del lema comtiano de Orden y progreso; la interpretación dominante de los términos entendía el progreso como crecimiento económico y modernización, y el orden como la fijación de las condiciones de tranquilidad en las cuales debía encontrarse el pueblo para permitir la proyección del progreso sin pausa.

Ligadas a estas ideas estaba la discusión sobre la civilización frente a la barbarie, que se remontaba a la dicotomía planteada por Domingo Faustino Sarmiento, de acuerdo a la cual, gauchos, negros y aborígenes eran bárbaros, personas incultas incapaces de apreciar las ventajas de una vida social fundadas sobre los principios liberales que garantizaban el camino hacia el progreso. Sostenían por ello la necesidad de eliminar la barbarie (mediante el orden) y afianzar la civilización trayendo población europea (para entrar en las vías del progreso).

1 "Esto consiste en creer que las generaciones que se suceden durante siglos en un territorio se transmiten en una sustancia invariable. Y que esta evolución, cuyos aspectos seleccionamos retrospectivamente de forma que nos percibamos a nosotros mismos como su desenlace, era la única posible, representaba un destino. Proyecto y destino son las dos figuras simétricas de la ilusión de la identidad nacional" (Balibar, 1988, pp. 135-136). 
no dan cuenta del proceso que subyace al mismo: entre las causas más mencionadas se encuentra la epidemia de fiebre amarilla que diezmó a la población de bajos recursos de Buenos Aires que no pudo refugiarse en las afueras de la ciudad, el reclutamiento forzado de hombres negros para luchar en las primeras líneas de batalla en las guerras de la independencia, las altas tasas de mortalidad y bajas tasas de natalidad debido a las malas condiciones en que vivían los afroargentinos.

Sin embargo, esta declinación de la población afro, fue artificialmente desaparecida por medio de las estadísticas oficiales para acercarse a la tan anhelada homogeneidad poblacional blanca. Reid Andrews (1989), historiador norteamericano interesado en la población afroporteña del siglo XIX da cuenta de una declinación armada artificialmente a partir de los censos nacionales. A través de la instauración de la categoría trigueño que aglutinaba a los negros-pardos y morenos, y que luego pasó a disolverse en la categoría Blanco.

No obstante, son variadas las evidencias que contradicen las afirmaciones sobre su desaparición, entre ellas, su frecuente aparición en fotografías, la continua actividad de la comunidad a través de sus periódicos y sociedades de ayuda mutua, muestras de que eran más los esfuerzos por hacerlos desaparecer antes de que ello realmente sucediera ${ }^{1}$.

Esto se debe a que los afroargentinos permanecieron, pero dejaron de ser negros para llegar a ser "ciudadanos argentinos" e incorporados a una gran masa de población que pasó a ser definida como una "nación blanca". Por eso, al igual que los indígenas, su condición diferencial quedó vinculada a categorías como "marginación" o "pobreza", pasando a constituir parte de la clase subalterna de una nación homogénea étnicamente. La gran afluencia de inmigrantes europeos desde el último cuarto del siglo XIX contribuyó a dar una apariencia de "realidad demográfica" a lo que no era otra cosa que una elaboración identitaria ${ }^{2}$.

Las diferencias étnicas se iban diluyendo en la percepción colectiva consolidando en su lugar un tipo de prejuicio social por el cual el color oscuro se asociaba, no a una distinción racial, sino a la pertenencia a las capas socioeconómicas inferiores ${ }^{3}$.

1 Para mayor información ver Reid Andrews, George (1989). Los Afroargentinos de Buenos Aires. Ediciones de la Flor, Buenos Aires. pp. 113.

2 Como afirma Reid Andrews (1989), el orgullo racial ha llegado a formar parte del verdadero núcleo de la identidad argentina y en especial, de la identidad porteña, produciendo errores como el hablar de la desaparición de los negros de Buenos Aires, cegándolos a la realidad que los rodeaba y respecto de las evidencias que contradecían directamente sus declaraciones.

"Los matices de los colores de la piel se van a trasformar paulatinamente en el indicador visible de una diferencia de naturaleza de las "sangres", invisibles portadoras de cualidades hereditarias superiores e inferiores. Las teorías racialistas y los racismos ideológicos de los siglos XIX y XX van a repensar estas representaciones, temores y esquemas, y reelaborarlos en el marco de un proyecto positivista, de fundación de la "ciencia del hombre", presuponiendo no solamente la existencia de clasificaciones naturalistas de las "razas humanas" sino también la descripción científica de sus caracteres distintivos y sus aptitudes respectivas" (Taguieff, 2002, p. 27).

Para citar este artículo:
Kleidermaster, G. (junio, 2012). Migraciones subsharianas a Argentina: interacción, integración o exclusión. Ánfora, 19 (32), 91-116. Universidad Autónoma de Manizales. ISSN 0121-6538. 
De este modo la raza (al igual que lo étnico) que habían sido categorías de identificación en el momento formativo del Estado-nación argentino, fueron dejadas de lado para resaltar otras categorías de identificación como la nacionalidad y la clase social, sirviendo de salvoconducto para quienes portaban un color de piel incriminador.

En este período nace la expresión Crisol de Razas' ${ }^{1}$, cuyo objetivo sería generar la representación hegemónica de un ser nacional que nace con la fundación de la nación, y es, en este sentido, un ser nuevo, esculpido por el Estado, a través de la escuela, la salud pública, y el servicio militar. La argentinidad es entonces concebida como una forma de etnicidad unitaria (Segato, 2007).

Como menciona Castro Gómez (2000) "El proceso de la civilización arrastra consigo un crecimiento del umbral de vergüenza porque se hacía necesario distinguirse claramente de todos aquellos estamentos sociales que no pertenecían al ámbito de la civitas que intelectuales latinoamericanos como Sarmiento venían identificando como paradigma de la modernidad"(Castro, 2000, p. 150).

\section{Metodología}

Dado que, como se dijo arriba, el racismo y la discriminación están desprestigiados, nadie se reconoce a sí mismo en esos términos, el discurso -oficial o privado- tiende a negar las prácticas cotidianas, expresadas en mensajes, enunciados y acciones, que de hecho imponen y reproducen modalidades de segregación y rechazo a vastos sectores de la población. Como observa Margulis (1997), el retroceso de la legitimidad del racialismo y la adopción de legislaciones antidiscriminatorias modificaron en parte las modalidades de expresión del racismo. Pero si bien la expresión directa, la hostilidad declarada o la discriminación abierta han retrocedido en algo, las desigualdades y las estratificaciones raciales persisten.

Es por ello que retomo el concepto de "racismo encubierto" planteado por De Rudder y Poiret (2000), el cual consiste en manifestaciones no explícitas, indirectas y no violentas de racismo que se desarrollan en un contexto de reflujo y de prohibición formal de sus formas de expresión explícitas, directas y ofensivas. Se caracteriza por tomas de posición conformistas, y una tendencia al statu quo (no hacer nada para mejorar la situación de los minoritarios).

Como mencionara anteriormente, en Argentina la legislación no impone formas evidentes de discriminación, no hay normas formalizadas que impongan a algunas clases de habitantes menores derechos. El discurso tiende a negar el racismo y la dis-

1 Dicha expresión también es analizada por Lea Geler (2004), entendida dentro del proceso de construcción del Estado Nación, donde las élites crean discontinuidades interiores generando jerarquías y tensiones, fundamentadas a partir de criterios raciales. 
criminación porque están mal vistas. Sin embargo, el racismo como ideología funciona en dos niveles: el de las acciones cotidianas y su interpretación, y en la negativa a reconocer el racismo o asumir la responsabilidad de éste.

Para poder advertir este racismo en la ciudad de Buenos Aires, se adoptó una metodología cualitativa en la que, a partir de la elección de un barrio de la ciudad de Buenos Aires, mediante observaciones y entrevistas con inmigrantes africanos y con la población "nativa", se intenta analizar, a través de su discurso, los imaginarios que circulan en ellos.

Esto se debe a que se parte de la noción de racismo cotidiano propuesta por Essed (1991), quien entiende cotidianidad desde el punto de vista de las categorías y las relaciones sociales vigentes en la vida cotidiana y desde las características de la misma para comprender el racismo como un proceso que se lleva a cabo en la vida diaria. "Las prácticas cotidianas están presentes y se reproducen a través de las situaciones cotidianas, que reposan en relaciones de raza, etnicidad, clase y género, lo cual conduce a la noción de racismo cotidiano" (p. 144).

Por lo tanto, el racismo cotidiano es la integración del racismo a las situaciones cotidianas mediante prácticas que activan situaciones de poder subyacentes. "En una sociedad racista, la raza y la etnicidad pueden ejercerse a través de cualquier relación social, cuando reconocemos las dimensiones raciales o étnicas dentro de las relaciones particulares, resulta entonces posible hablar del racismo cotidiano como la activación situacional de dimensiones raciales o étnicas dentro de relaciones particulares, de manera tal que refuerza la desigualdad racial o étnica y contribuye a nuevas formas de desigualdad racial y étnica" (Essed, 1991, p. 147).

Ahora, elección del barrio de Flores se debe a dos razones: en primer lugar, porque al tratarse de un estudio exploratorio, se restringe a una zona que de fácil acceso y representativa de las situaciones que pueden presentarse en la vida cotidiana, y por otro lado, que presentase una cantidad considerable de interacciones entre los inmigrantes africanos que venden bijouterie en la vía pública y numerosos vendedores ambulantes y comercios donde poder observar dichas interacciones.

Flores es un barrio tradicional, hoy de clase media, ubicado en el centro geográfico de la Ciudad Autónoma de Buenos Aires. Está comprendido por las calles Av. Gaona, Tte. Gral. Donato Álvarez, Curapaligüe, Av. Directorio, Av. Castañares, Curapaligüe, Camilo Torres y Tenorio, Av. Riestra, Av. Perito Moreno, Av. Castañares, Lacarra, Av. Luís J. Dellepiane, Au. 25 de Mayo, Portela y Cuenca. Limita con los barrios de Villa Santa Rita y Villa Mitre al norte, Caballito y Parque Chacabuco al este, Nueva Pompeya y Villa Soldati al sur, y Parque Avellaneda y 104 Floresta al oeste.

Para $\quad$ Kleidermaster, G. (junio, 2012). Migraciones subsharianas a Argentina: interacción, integración 
La observación para el presente trabajo se realizó en las Avenidas Rivadavia (desde Plaza Flores hasta Donato Álvarez) y Avellaneda (Desde Nazca hasta Campana), por tratarse de dos polos comerciales con cuantiosos transeúntes y vendedores ambulantes así como de una dinamizada zona comercial de la ciudad.

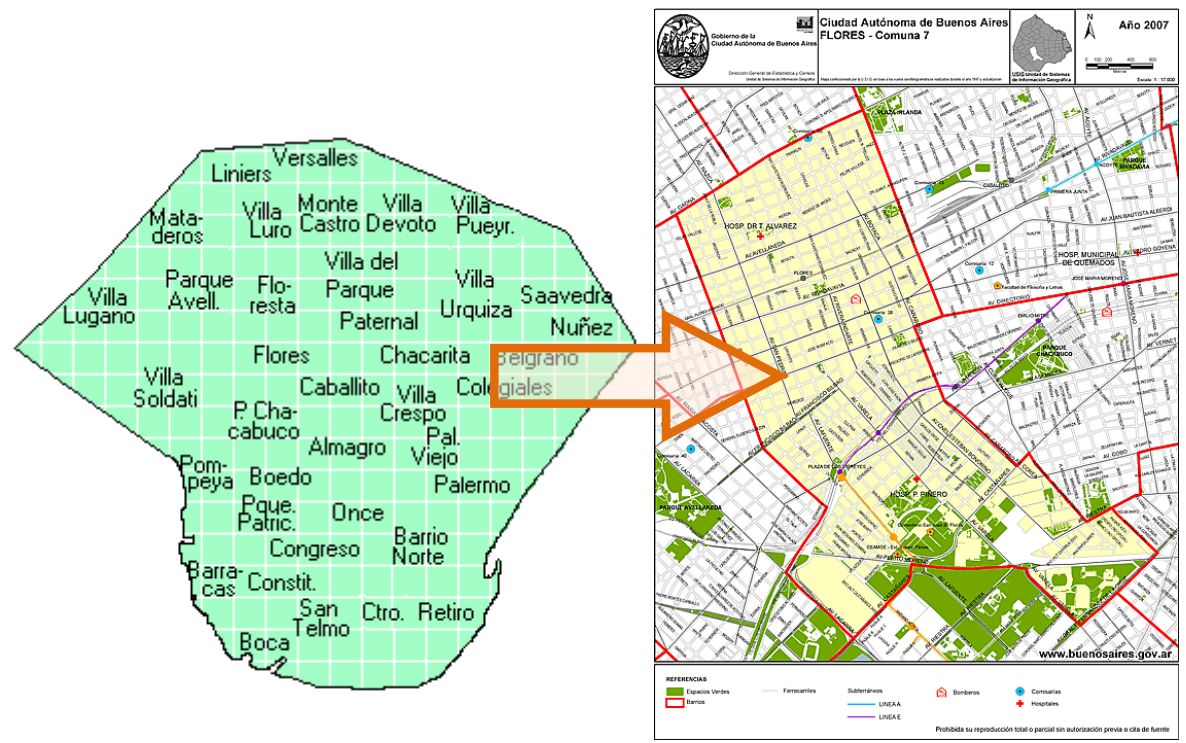

En cuanto a los discursos, el análisis se efectuó sobre la base de 40 entrevistas semi estructuradas, muchas de ellas realizadas de manera informal, dado que se trata de un estudio exploratorio. Consecuentemente, consistió en un primer acercamiento a la población que trabaja en dicho barrio, durante su jornada laboral, por lo cual fueron producto de interrupciones varias. No obstante ello, se realizaron 30 entrevistas a la población que denomino "nativa", es decir, argentinos que trabajan en comercios y en la venta ambulante en dicha zona, y 10 a vendedores ambulantes provenientes de la reciente inmigración del áfrica subsahariana para analizar cuáles son sus sensaciones y percepciones acerca de su situación y contacto con la población de dicho barrio.

El análisis se realizó en base a la Teoría Fundamentada o Grounded Theory (Glaser y Strauss, 1967), en base a una grilla de codificación cualitativa ${ }^{1}$.

1 La teoría fundamentada fue desarrollada por dos sociólogos, Glaser y Strauss, en base a una investigación donde se desarrolló el método comparativo constante, que más tarde fue conocido como la teoría fundamentada. En lugar de comenzar con una hipótesis, el primer paso es la recopilación de datos, a través de una variedad de métodos. De los datos recogidos, los puntos principales están marcados con una serie de códigos, que son extraídas del texto. Los códigos se agrupan en conceptos similares con el fin de hacerlas más viables. A partir de estos conceptos, las categorías se forman, que son la base para la creación de una teoría. 


\section{Algunos imaginarios nativos}

Para entender las relaciones sociales, en las que se insertan las manifestaciones discriminatorias, hay que prestar especial atención a los discursos. La elección de esta metodología se apoya en la observación de Mármora (2004) para quien el prejuicio anti-inmigratorio se genera, consolida y justifica mediante rumores y consignas donde se acusa al extranjero de provocar efectos negativos o portar virtudes siniestras, sobre todo en momentos de crisis económicas o sociales (Mármora, 2004).

\section{Resultados}

Debido fundamentalmente a la pérdida de legitimidad de las ideologías racistas, así como de las leyes anti-discriminación surgidas en los últimos tiempos, no sólo en nuestro país, sino en un plano internacional, los entrevistados intentan cuidar sus palabras para no parecer "discriminadores": muchos dicen que los derechos tienen que ser para todos. Sin embargo, al responder a las preguntas referidas a los problemas de sobrepoblación en los hospitales y escuelas, así como también muchas respecto del desempleo, consideran que el país no está capacitado para seguir recibiendo migrantes: muchos de ellos hablan de "cupos" o "cuotas" que el país debería establecer, para, una vez superados, cerrar las fronteras. “(...) los protegen, no como en Europa. No son tantos como para que contaminen la raza, sin ser racista pero creo que habría que controlar más el ingreso (...) Habría que haber un seguimiento de los inmigrantes que hay, un control" (Hombre, 56 años, diariero).

$\mathrm{Al}$ indagar acerca de las representaciones que se tienen respecto de los migrantes africanos, sorprendió que la respuesta surge por comparación a los migrantes limítrofes (bolivianos y peruanos especialmente), quienes ocuparían el lugar de "otros" radicales. Su figura concentra diversos prejuicios presentes en la sociedad, muchos de ellos producto de los discursos del Estado y los medios de comunicación, difundidos principalmente en la década del 90. Su imagen, portadora de los males sociales, a quien se atribuyen los disturbios, el "andar en cosas raras" y vivir en forma maliciosa, se compara con la del africano, que parece ser mas "aceptable", sin por ello quedar exento de prejuicios: "creo que son mejores gentes que los peruanos y bolivianos que vienen acá. Si no hacen nada con ellos, menos lo van a hacer con estos". (Mujer, 65 años, Jubilada)

En general los imaginarios que los nativos mantienen frente a estos "otros" tienen que ver con la asignación de cualidades negativas y sospechas respecto a su actividad de subsistencia. Dado que su inserción laboral es fundamentalmente marginal, ligada a la venta ambulante de Bijouterie, el desconocimiento frente a esta población y a su actividad laboral, sumadas al distanciamiento frente a ellos, funcionan como un importante productor de prejuicios, donde se les asigna una connotación ilegal a su actividad. Existe una situación perceptible acerca de cierto desconocimiento sobre los "otros"

Para citar este artículo:
Kleidermaster, G. (junio, 2012). Migraciones subsharianas a Argentina: interacción, integración o exclusión. Ánfora, 19 (32), 91-116. Universidad Autónoma de Manizales. ISSN 0121-6538. 
que genera temor en muchos autóctonos. Se dice que la discriminación y el racismo son hijos del miedo y la ignorancia:

(...) manejan mucho el oro líquido. Lo funden y lo usan líquido en botellas de coca cola, y lo vuelven a re vender, ¿'vos sabías eso? (Hombre, 45 años, vendedor de diarios)

(...) son medios artesanos, trabajan con cosas que hacen con las manos. Me han ofrecido trabajo artesanal y relojes, deben conocer el material, ellos saben y la marca que venden son de primera línea pero adulteradas. La garantía no es la misma (Hombre, 60 años, boletero línea de colectivo)

Sin embargo, un rasgo que gran parte de los entrevistados resaltan, es el de su extrema responsabilidad para con el trabajo, que, según se infiere, se vincula al fuerte legado de la esclavitud que relaciona al negro con el trabajo arduo y de extensas jornadas:

Son como los bolivianos, laburan a pleno, sin descanso. De lunes a domingo todo el día (Mujer. Empleada de comercio de indumentaria femenina. 25 años)

Son súper laburadores, están todo el día acá, parados en la calle, abajo del sol, no se mueven (...) (Mujer, 36 años, Responsable de local) ${ }^{1}$.

Asimismo, se desprende de sus discursos la responsabilidad en la falta de relación, en algunos casos producto de su falta de interés. Sin embargo, está también presente la atribución de la culpa al colectivo "por ser cerrado y no querer relacionarse". Ello contribuye a la generación de estereotipos, principalmente en relación a su presencia, producto del desconocimiento y en representaciones que han quedado en el imaginario de la población en relación a los afro-argentinos durante la colonia.

Como se menciona antes, los afroporteños, durante la esclavitud y en años posteriores a su abolición, eran vistos como "traidores", "ociosos", "faltos de educación", "sucios" (Rodríguez Molas, 1961). Tales calificativos parecen reproducirse en ciertos discursos actuales:

Por ahí no tienen mucha higiene, porque no tienen un buen nivel de vida (...) "vienen con cada olores... falta desodorante(...)" (Mujer, 25 años, empleada de comercio)

Su higiene deja mucho que desear, la manguera de bomberos mal no les vendría (Hombre, 48 años, vendedor ambulante).

1 "Laburar" es un término utilizado como sinónimo de "trabajar" en lunfardo (jerga originada y desarrollada en la Ciudad de Buenos Aires y el Gran Buenos Aires debido en gran medida a la millonaria inmigración europea, principalmente italiana y española) ocurrida principalmente entre los años 1870 y 1960. Originariamente esta jerga era empleada por los delincuentes y pronto lo fue por la gente de las clases baja y media-baja. Parte de sus vocablos se introdujeron posteriormente en la lengua popular y se difundieron en el castellano de la Argentina y Uruguay. Sin embargo ya a inicios del siglo XX el lunfardo comenzó a difundirse entre todos los estratos y clases sociales ya sea por la habitualidad de su uso, ya sea porque era común en las letras de tango. 
No son muy limpios, porque están en África, no es Petersburgo, son medio sucios (Hombre, 36 años, comerciante).

Como se desprende del análisis de las entrevistas, parece haber una adjudicación de características que relacionan sujetos con naciones en una jerarquización donde habría "naciones ricas - sujetos deseables" frente a "naciones pobres- sujetos indeseables". (Pottilli, Silberstein y Tavernelli ,2009).

Es esta imagen de un África pobre, donde vivirían en la total miseria, lo que justificaría sus malas condiciones de vida aquí, donde siempre serían superiores a las existentes en su lugar de origen:

Acá están mejor que allá. En una situación que tal vez no sea la adecuada pero mejor que allá, si no, no se irían para vivir en una manera precaria (hombre, empleado de comercio, 30 años.

Siempre van a vivir mucho mejor que en África. Viven en la indigencia total, pero no tienen que pagar un alquiler ni monotributo (...) Por lo menos tienen agua (Hombre, Dueño de puesto de diarios, 48 años).

Algo mejor de lo que vivían allá porque no tienen igualdad porque la gente los discrimina, la integración cultural cuesta bastante y más a ellos (mujer, 25 años, empleada de negocio).

Se codifica al migrante africano como sujeto inferior, trasgresor, ilegal, en sujeto desviado de la normalidad, y como consecuencia de ello, justificadamente excluido,

No tiene posibilidad de hacerse de un lugar, de elegir libremente su lugar, el suyo es el lugar de su origen nacional, de sus raíces, la distancia que él mantenga con su origen no cuenta para el nativo. Esta mirada sobre el otro no sólo detecta las diferencias, sino que hace de ellas un sistema de jerarquías, de inclusión-exclusión. (Cohen, 2009).

Hay una jerarquía presente en la sociedad, donde el diferente es inferior al nativo, y no sólo eso, sino que también es peligroso, porque usurpa lo que no le corresponde y por lo tanto está bien limitarlo, ya sea en su inserción laboral, en sus oportunidades educativas o en su ubicación geográfica, porque es en legítima defensa. Es una forma de discriminación y de establecer relaciones desiguales, relaciones donde el nativo es el dominante y tiene el derecho a decidir dónde debe asentarse la población inmigrante, y también, en qué trabajos debe insertarse.

En este sentido, parece natural que el migrante tenga una inserción precaria en el mercado laboral. Con base en las entrevistas realizadas con "nativos", manifiestan su convencimiento respecto a su derecho a conseguir mejores trabajos que los no nacionales (en particular, los migrantes no deseados, con características fenotípicas que los colocarían en inferioridad racial), como una perpetuación del razonamiento incorporado por la colonia, definido como racializacion de las relaciones de clase. "Hay una ley que los protege en la venta ambulante. No se los puede tocar. Es una competencia desigual, vos no podés 108 competir con un tipo que no paga monotributo ${ }^{1}$ ni nada" (Hombre, 45 años, diariero).

Para citar este artículo:
Kleidermaster, G. (junio, 2012). Migraciones subsharianas a Argentina: interacción, integración o exclusión. Ánfora, 19 (32), 91-116. Universidad Autónoma de Manizales. ISSN 0121-6538. 
Asimismo, vinculado al concepto de racismo encubierto, donde se considera al mismo, no sólo como una actitud activa, sino además como la pasividad frente a relaciones de desigualdad, esta inacción de la población frente al reconocimiento de las dificultades a las que se enfrentan los migrantes africanos, que surge del análisis de las entrevistas, deja entrever situaciones de discriminación y racismo que se hacen más visibles frente a la indagación acerca de lo que se espera que haga el gobierno, donde la mayor parte de los entrevistados se centra en el pedido de acciones hacia la población nacional, marcando una frontera entre "ellos" y "nosotros", pero también entre el individuo y el resto de la sociedad que sería más racista:

(...) en otros lugares no los toman. Son como terroristas, tienen visas de todos lados, pero no los toman por el color y tema de estudios (Hombre, 26 años, empleado en comercio de indumentaria).

(...) no les van a dar trabajo acá porque es un país racista (...) (Mujer, 32 años, empleada en comercio de indumentaria)

(...) no tienen la posibilidad de que los comercios y las empresas los puedan contratar por la discriminación, es algo social (...) (Mujer, 29 años, encargada de negocio de indumentaria masculina).

Sin embargo, en el momento de reclamar acciones frente al gobierno, en la gran mayoría de los casos se solicitaron acciones para la población nacional. Si bien muy pocos solicitaron el cierre de fronteras o el control de la inmigración, que sí aparece claramente como un discurso anti-discriminatorio, el hecho de que tampoco se soliciten medidas a favor de la situación que anteriormente se reconoce como desfavorable plantea un serio problema para su integración.

Pienso que todos los que vienen de afuera están mejor que nosotros, porque no pagan impuestos y no se les exige nada. Deberían ponerlos en las mismas condiciones, con las mismas obligaciones que tenemos todos. Que les cobren los impuestos porque son iguales a nosotros. ¿Porque yo voy a pagar los impuestos si el otro no paga nada? (Hombre. 60 años, encargado en Puesto Diarios).

Que los ubique en alguna feria artesanal. Bajo techo, y que paguen algún impuesto, porque éstos no pagan nada. Que tengan un costo, aunque estén en la vereda (Hombre, 60 años, Boletero).

Debería empezar por nosotros primero, a mí la jubilación no me alcanza. Deberían empezar por los argentinos pero hay privilegios porque hay comunidades migrantes que votan (Mujer de 65 años, Vende productos en la calle. jubilada).

Pareciera ser que sólo los individuos que forman parte de la nación argentina fueran pasibles de recibir políticas del gobierno que los favorezca, mientras que "los otros",

1 El Monotributo es un impuesto que concentra en un importe fijo la seguridad social y un valor impositivo. La obligación de pago es mensual. El ingreso de las sumas establecidas para los distintos componentes es de carácter obligatorio. 
los inmigrantes no deseados que no forman parte del "pueblo", deben ser controlados, asignados a determinados lugares donde trabajar, y, en caso de sobrepasar alguna "medida cuantificable de la población que puede tolerarse en el país", ser enviados a sus lugares de origen (Cohen, 2009).

Resulta interesante, al respecto, el análisis de Balibar (1988) acerca de la construcción de esta idea de pueblo como producción artificial que denomina "etnicidad ficticia", es decir, la comunidad formada por el Estado nacional, en el sentido de efecto institucional de fabricación. “(...) ninguna nación posee naturalmente una base étnica, pero a medida que las formaciones sociales se nacionalizan, las poblaciones que incluyen, o que dominan quedan 'etnificadas', es decir, quedan representadas en el pasado o en el futuro como si formaran una comunidad natural, que posee por sí misma una identidad de origen, de cultura, de intereses, que trasciende a los individuos y las condiciones sociales" (Balibar, 1988, p. 149)1.

Oteiza, Novick y Aruj (1997) plantean que el inmigrante no deseado, el "otro", no es visto como un ser humano persona, sujeto de los mismos derechos fundamentales que "nosotros", es un excluido excluible y a la vez una amenaza, pasible de ser expulsado, o perseguido de diversas formas por un Estado que puede actuar frente a él sin los límites de ningún tipo de legalidad.

La presencia del migrante no es rechazada, es aceptada pero bajo condiciones de dominación, donde las minorías involucradas no tienen el derecho a elegir y participar libremente de ciertos espacios. El prejuicio, la discriminación y la segregación étnica y nacional son manifestaciones distintas de la exclusión social, lo que permite controlar al otro, circunscribiéndolo a un espacio aislado y estigmatizado. Se trata de limitar, acotar, obstaculizar, es un sujeto limitado en sus posibilidades de participación social, política y económica.

\section{Algunas percepciones de los propios migrantes}

Para finalizar, y retomando las nociones planteadas por Essed (1991) para estudiar el racismo, es importante observar las relaciones sociales vigentes en la vida cotidiana, ya que es en dichas situaciones donde las prácticas racistas se activan, y donde el racismo actúa, se expresa y se consolida a través de las relaciones de poder.

Se puede entender, entonces, el racismo cotidiano como la activación situacional de relaciones raciales o étnicas, las cuales pueden ejercerse en cualquier relación social siempre que se activen dichas dimensiones, de manera tal que refuerza la desigualdad y/o contribuye a nuevas formas de manifestarla.

1 Ésta etnicidad se produce por medio de la lengua y la raza, eso es lo que permite representarse al pueblo como una unidad absolutamente autónoma. Es decir, lo que permite vincular el carácter nacional con el pueblo, que, como vimos al comienzo del trabajo, fue parte de un arduo proceso de homogeneización y fabricación de un imaginario que perdura hasta nuestros días. (Balibar, 1988:149) 
Para ello, es importante analizar el discurso de la población nativa para desentrañar los imaginarios que presentan frente a la población inmigrante. Pero a la vez, son de central importancia los discursos de los inmigrantes africanos donde describen sus experiencias y dan cuenta de estas relaciones, mediadas por relaciones de poder con base en características fenotípicas. Lo que interesa son las interpretaciones compartidas de la realidad social.

El diálogo con ellos presentó ciertas dificultades, las cuales se desprenden de las situaciones de violencia y racismo que viven en sus vidas cotidianas. La desconfianza frente a las preguntas de un nativo, al cual atribuyen el rol de control migratorio o policial, fue uno de los principales impedimentos.

No obstante, surgieron discursos interesantes, donde manifestaron que una de las principales dificultades que encuentran se centra en la obtención de viviendas:

A. (...) a veces hay problema de conseguir donde vivir ¿viste en las casas donde viven los chicos? (...) no es que no tenen de que pagar un depto o algo así pero el tema es que no los alquilan los deptos por tema de documentación o garantía o algo así (...). yo entante muchas veces pero si no tenés eso o una persona de confiencia que te alquila su depto no puede

B. ¿y con los hoteles o pensiones?

A. Ahí si te alquilan, ¿viste donde viven los chicos? (...) a veces si a veces no, alguno te alquilan y te querían mandar o dar límites (...) si era las reglas también no lo aplicaban con todos" (Entrevista a migrante Senegalés, Hombre, 27 años).

Los africanos no alquilamos casa sino habitación. Yo fui, estaba el cartel y cuando fui me dijeron que ya estaba ocupada, le pedí a una chica blanca que vaya y le alquilaron... (Inmigrante de Ghana, Hombre, 22 años).

En segundo lugar, se plantea la problemática laboral donde en su amplia mayoría manifiestan querer realizar otra actividad, pero se sienten inseguros con el idioma y creen que no los tomarían en otros puestos. En general, su mayor dificultad se debe a la policía, quien no los deja realizar la venta ambulante1. "Antes vendía en Palermo pero policía molestaban mucho, ahora trabajo en Flores pero después de las tres de la tarde cuando ya no hay policía. El año pasado, para navidad me sacaron las cosas y fui a reclamar pero no devolvieron" (Inmigrante de Sierra Leona, hombre, 24 años).

Los inmigrantes son conscientes de que no están en su país, y la sociedad se lo hace sentir, por eso intentan focalizarse únicamente en el trabajo, y, como ellos dicen "no buscar problemas": "Cuando la gente grita cosas no puedo decir nada porque se arma revuelo, llega la policía y siempre defiende al argentino" (Inmigrante de Ghana, 23 años, Hombre).

1 Hay un juicio iniciado por tres senegaleses con el patrocinio de un grupo de abogados pertenecientes al CELS (Centro de Estudios Legales y Sociales), en contra de la Fiscalía de la Ciudad de Buenos Aires, alegando que detiene arbitrariamente a los vendedores senegaleses, decomisando su mercadería, reteniendo su documentación y actuando de manera discriminatoria. Tras numerosas alegaciones, el fallo resultó favorable para el colectivo Subsahariano. 
El trabajo es el núcleo articulador entre la esfera económica y social, la discriminación se da por su inserción laboral segmentada y vulnerable. De esta manera la diversidad se homologa a desigualdad: de oportunidades, de participación en el mercado de trabajo, de acceso a servicios de salud, educación o vivienda, de derechos, etc.

Esta diversidad inferioriza al otro, se reconocen señales que lo desacreditan, fundando relaciones de dominación entre nativos y extranjeros, que cristalizan un mundo que se divide entre los incluidos y los excluidos.

Por un lado hay un actor social favorecido en esta distribución social y económica de roles, portador de una identidad nacional y étnica reconocida, y por el otro hay un actor social desempeñando el rol de dominado, culpabilizado.

\section{Conclusiones}

Los discursos demuestran que efectivamente hay un espacio de conflicto donde el migrante "molesta”, resulta "extraño", no sólo por su condición nacional, sino, aunque no se acepte, por su condición racial.

$\mathrm{Al}$ parecer, las fuertes herencias coloniales siguen vigentes en los imaginarios porteños en relación a la manera de representarse al "otro" de piel más oscura. Centurias de coloniaje han dejado fuertes improntas en nuestra manera de representarnos las características de lo africano, como un ser inferior. Asimismo, los idearios racistas incorporados por las élites dominantes que dieron forma al Estado-nación argentino, siguen también vigentes para la representación de un pueblo homogéneamente blanco, donde los fenotipos más oscuros son asociados a los estratos inferiores de la sociedad como un lugar legítimo al cual deben pertenecer.

Como observa Cohen (2009), marcar un lugar para el otro, controlar sus acciones y su lugar en la sociedad implica también imponerle un orden, en donde está incluido, -en una sociedad que se considera abierta, multicultural, multiétnica, "crisol de razas"-, pero desde un lugar de exclusión.

El trabajo es la principal manera de vincularse con el resto de la sociedad, donde se establecen vínculos para la integración. En Argentina consiste también en una fuente de derechos (aportes jubilatorios, seguridad social), etc. El hecho de estar confinados a una actividad marginal, implica un fuerte limitante para una integración real, formal y social.

Este lugar que se les pauta a los senegaleses, tanto en el mercado laboral, como en el sistema de salud, o mismo en la geografía de la ciudad, se enmarca dentro de una relación racial, de dominación con base en los rasgos fenotípicos particulares, que no son los deseados por el imaginario nacional. Dicha dominación se reproduce en la vida cotidiana a través de relaciones raciales, las cuales se propuso desentrañar este trabajo. 
Las ideas de Sarmiento, sobre la superioridad de las poblaciones blancas y Europeas, en contraste con las indígenas y negras, sinónimo de barbarie y atraso, siguen vigentes en gran parte del imaginario argentino, más aún en la constitución nacional, nuestro imaginario, inclusive en nuestra constitución que promueve la inmigración de origen europeo .

Si bien se ha planteado como un artículo con carácter exploratorio, un intento por observar y conceptualizar ciertos imaginarios que los argentinos tienen respecto a la nueva corriente de inmigrantes subsaharianos. En el transcurso, nuevos interrogantes han aparecido, relacionados con problemáticas a las que se enfrentan diariamente la población afro. Asimismo, surgen preguntas por los discursos de los argentinos en el marco de políticas democratizantes, leyes anti-discriminatorias y una nueva legislación migratoria que parece ser más inclusiva.

Estas y otras cuestiones serán fuente de nuevas investigaciones que contribuyan a desentrañar una temática tan difícil de sacar a la luz como lo es el racismo y la discriminación en el marco de narrativas multiculturales y teorías que reafirman la no existencia de diferencias raciales.

\section{Referencias}

Balibar, E. (1988). La forma nación: historia e ideología. En Wallerstein, I. y Balibar, E. (1988). Raza, Nación y clase. Madrid: IEPALA.

Caggiano, S. (2008). Racismo, fundamentalismo cultural y restricción de la ciudadanía. Formas de regulación social frente a los inmigrantes en la Argentina. En Novick, S. (Ed.). Las migraciones en América Latina. Políticas, culturas y estrategias. Buenos Aires: Catálogos.

Castro, S. (2000). Ciencias sociales, violencia epistémica y el problema de la "invención del otro". En Lander, E. (Comp.) La colonialidad del saber: eurocentrismo y ciencias sociales. Perspectivas Latinoamericanas. Buenos Aires, Clacso, Consejo Latinoamericano de Ciencias Sociales.

Cohen, N. y Mera, C. (Eds.) (2005). Relaciones interculturales: experiencias y representación social de los migrantes. Buenos Aires: Antropofagia.

De Rudder, V. y Poiret, C. (2000). La desigualdad racista. Precisiones conceptuales y propuestas teóricas. En Hoffman, O. y Quintero, O. (Eds.). (2010). Estudiar el racismo. Textos y herramientas. Documento de Trabajo No8, México: Proyecto AFRODESC/EURESCL.

Essed, P. (1991). Hacia una conceptualización del racismo como proceso. En Hoffman, O. y Quintero, O. (Eds.). (2010). Estudiar el racismo. Textos y herramientas. Documento de Trabajo 8. México: Proyecto AFRODESC/EURESCL.

Frigerio, A. (2000). Cultura negra en el Cono Sur: Representaciones en conflicto. Buenos Aires: Ediciones de la Universidad Católica Argentina. 
Frigerio, A. (2002). Negros y blancos en Buenos Aires: repensando nuestras categorías raciales. Ponencia presentada en las jornadas Buenos Aires Negra: Memorias, representaciones y prácticas de las comunidades afro, Buenos Aires.

Frigerio, A. (2006). De la "desaparición" de los negros a la "reaparición" de los afrodescendientes: Comprendiendo la política de las identidades negras, las clasificaciones raciales y de su estudio en la Argentina. Paper presentado en el Seminario Internacional Los Estudios Africanos en América Latina: Herencia, Presencia y Visiones del Otro. Universidade Federal da Bahía.

Funes, P. y Ansaldi, W. (2004). Cuestión de piel: Racialismo y legitimidad política en el orden oligárquico latinoamericano. En Ansaldi, W. (ed.) Calidoscopio Latinoamericano (pp. 451-495). Buenos Aires: Ariel Historia

Geler, L. (2004). Negros, pobres y argentinos. Identificaciones de raza, de clase y de nacionalidad en la comunidad afroporteña, 1870-80. Revista Nuevo Mundo, Mundos Nuevos, 4.

Geler, L. (2007). ¡Pobres negros! Algunos apuntes sobre la desaparición de los negros argentinos. En García Jordan, P. (Ed.). Estado, religión y poder local en América Latina. Siglos XIX-XX. Barcelona: Publicacions i edicions de la universitat de Barcelona

Geler, L. (2010). Andares negros, caminos blancos: afroporteños, Estado y nación. Argentina a fines del siglo XIX. Rosario: Protohistoria Ediciones.

Glaser, B. \& Strauss, A. (1967). Discovery of Grounded Theory. Strategies for Qualitative Research. Sociology Press.

Goldberg, M. (1976). La población negra y mulata en la ciudad de Buenos Aires. Revista Desarrollo Económico, 16, (61), 75-99.

Goldberg, M. y Mallo, C. (1994). La población africana en Buenos Aires y su campaña. Formas de vida y subsistencia. Revista Temas de Africa y Asia 2, Publicación de la Facultad de Filosofía y letras, UBA.

Guillaumin, C. (1992). Una sociedad en orden. Sobre algunas de las formas de la ideología racista. En Hoffman, O. y Quintero, O. (Eds.) (2010). Estudiar el racismo. Textos y herramientas. Documento de Trabajo No8, México: Proyecto AFRODESC/EURESCL.

Maffia, M. (1986). La inmigración caboverdeana hacia la Argentina. Análisis de una alternativa. Revista Trabalhos de Antropología e Etnología, 25, 191-207.

Maffia, M., Ottenheimer, A. y Zubrzycki, B. (2007). Nuevos inmigrantes africanos en Argentina. En Actas de las XI Jornadas Interescuelas - Departamentos de Historia. Tucumán.

Margulis, M. (1999). La segregación negada. Buenos Aires: Biblos.

Mármora, L. (2004). Las politicas de migraciones internacionales. Buenos Aires: Paidós-OIM.

Memmi, A. (1994). El racismo. Definiciones. En Hoffman, O. y Quintero, O. (Eds.) (2010). Estudiar el racismo. Textos y herramientas (pp. 53-72). Documento de Trabajo 8, México: Proyecto AFRODESC/EURESCL.

114 Morner, M. (1970). La mezcla de razas en la historia de América Latina. Buenos Aires: Paidos.

Para citar este artículo:
Kleidermaster, G. (junio, 2012). Migraciones subsharianas a Argentina: interacción, integración o exclusión. Ánfora, 19 (32), 91-116. Universidad Autónoma de Manizales. ISSN 0121-6538. 
Oteiza, E., Novick, S y Aruj, R. (1997). Inmigración y discriminación. Políticas y discursos. Buenos Aires: Grupo Editor Universitario.

Picotti, D. (1998). La presencia africana en muestra identidad. Buenos Aires: Ed. del Sol.

Picotti, D. (2001). El negro en la Argentina. Presencia y negación. Buenos Aires: Editores de América Latina.

Pottilli, J., Silberstein, Y. y Tavernelli, R. (2009). De la jerarquización de naciones a la clasificación de sujetos: representaciones que perpetúan un orden exclusor. En Cohen, N. (Coord.). Representaciones de la diversidad: trabajo, escuela y juventud. (pp. 30-65). Buenos Aires: Ediciones Cooperativas.

Quijada, M. (2004). De mitos nacionales, definiciones cívicas y clasificaciones grupales. Los indígenas en la construcción nacional argentina, siglos XIX a XXI. En Ansaldi, W. (Ed.). Calidoscopio Latinoamericano. Buenos Aires: Ariel Historia.

Quijano, A. (2000). Colonialidad del poder, eurocentrismo y América Latina. En Lander, E. (comp.). La colonialidad del saber: eurocentrismo y ciencias sociales. Perspectivas Latinoamericanas. Buenos Aires, CLACSO, Consejo Latinoamericano de Ciencias Sociales.

Reid, G. (1989). Los Afroargentinos de Buenos Aires. Buenos Aires: Ediciones de la Flor.

Rodríguez, R. (1957). El primer libro de entradas de esclavos negros en Buenos Aires. Revista de la Universidad de la Plata, 1.

Rodríguez, R. (1961). Negros Libres Rioplatenses. Revista de Humanidades. Buenos Aires, 1 (1).

Sarmiento, D. (1995). Conflicto y armonía de las razas en América. Conclusiones. En Zea, L. (Ed.). Fuentes de la Cultura Latinoamericana. (pp. 401-411). México: Fondo de Cultura Económica.

Scheuss de Studer, E. (1958). La trata de negros en el Río de la Plata durante el siglo XVIII. Buenos Aires.

Segato, R. (2007). La Nación y sus Otros. Raza, etnicidad y diversidad religiosa en tiempos de Políticas de la Identidad. Buenos Aires: Prometeo.

Solomianski, A. (2003). Identidades secretas: La negritud argentina. Buenos Aires: Beatriz Sarlo Editora.

Stavenhagen, R. (2000). Conflictos étnicos y Estado nacional. México: Siglo XXI Editores.

Taguieff, P. (2002). Introducción al libro El color y la sangre. Doctrinas racistas a la francesa. En Hoffman, O. y Quintero, O. (2010). Estudiar el racismo. Textos y herramientas. México: Proyecto AFRODESC/EURESCL. Documento de Trabajo 8, 21-35.

Vela, M. (1999). Los afroamericanos en el imaginario de algunos intelectuales argentinos del siglo XIX. En Cáceres Gómez, R. (Ed.). Rutas de la Esclavitud en San José. Costa Rica: Editorial de la Universidad de Costa Rica. 
Vela, M. (2001). Historia y actualidad de los estudios afroargentinos y africanos en la Argentina. En Picotti, D. (Coord.). El negro en la Argentina. Presencia y negación (49-62). Buenos Aires: Editores de América Latina.

Villalpando, W. (2005). Hacia un plan nacional contra la discriminación: La discriminación en la Argentina. Buenos Aires: Inadi.

Wieviorka, M. (2009). El racismo: una introducción. Barcelona: Gedisa.

Zubrzycki, B. y Agnelli, S. (2009). Allá en África, en cada barrio por lo menos hay un senegalés que sale de viaje. La migración senegalesa en Buenos Aires. Cuadernos de Antropología Social, 29. 